\title{
Nonhealing Ulcerative Hand Wound
}

\author{
Imelda Muller, MD; Tatyana Yetto, MD
}

\begin{abstract}
Eligible for 1 MOC SA Credit From the ABD
This Photo Challenge in our print edition is eligible for 1 self-assessment credit for Maintenance of Certification from the American Board of Dermatology (ABD). After completing this activity, diplomates can visit the ABD website (http://www.abderm.org) to self-report the credits under the activity title "Cutis Photo Challenge." You may report the credit after each activity is completed or after accumulating multiple credits.
\end{abstract}
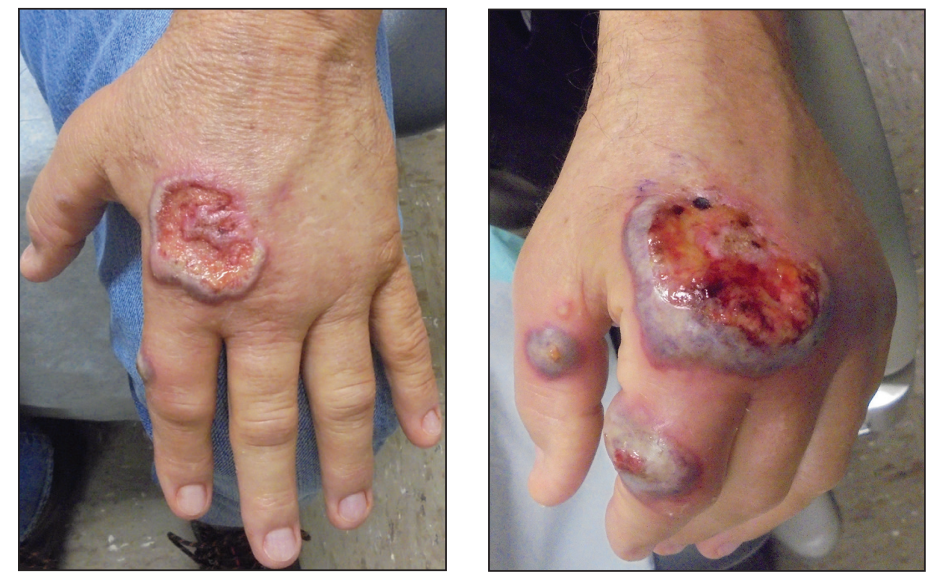
A 63-year-old man presented with an expanding wound on the dorsal aspect of the left hand after striking it on a wall. He sustained a small laceration that progressively became more edematous and developed a violaceous border. He presented to the emer- gency department the following day and was prescribed bacitracin with no improvement in the lesion. He returned to the emergency department after the symptoms worsened and was subsequently prescribed a 10-day course of oral trimethoprim-sulfamethoxazole (1600/320 mg) twice daily.

Physical examination at a follow-up visit 11 days after the initial injury revealed an expanding, $4.3 \times 5.0-\mathrm{cm}$, ulcerated wound with surrounding erythema and serosanguineous drainage (left). He was started on a 10-day course of amoxicillin-clavulanic acid (1750/250 mg) twice daily and underwent debridement the same day. On postoperative day 2 (13 days following the onset of symptoms), the wound had not improved, and 2 new $1-\mathrm{cm}$ bullae on the left first and second fingers had progressed (right). Erythrocyte sedimentation rate (33 mm/h [reference range, $0-10 \mathrm{~mm} / \mathrm{h}$ ]) and C-reactive protein (3.701 mg/dL [reference range, $0-0.747 \mathrm{mg} / \mathrm{dL}$ ]) were elevated; however, other laboratory studies, including a complete blood cell count, were within reference range. He remained afebrile, and a review of systems was normal. Punch biopsy specimens were obtained.

\section{WHAT'S THE DIAGNOSIS?}

a. blastomycosislike pyoderma

b. bullous cutaneous small vessel vasculitis

c. Mycobacterium marinum (fish tank granuloma)

d. necrotizing fasciitis

e. neutrophilic dermatosis of the dorsal hands

Dr. Muller is from the Navy Experimental Diving Unit, Panama City Beach, Florida. Dr. Yetto is from the Naval Hospital, Bremerton, Washington. The authors report no conflict of interest.

The views expressed herein are those of the authors and do not necessarily reflect the official policy or position of the Department of the Navy, Department of Defense, or US Government.

Correspondence: Imelda Muller, MD, Navy Experimental Diving Unit, 321 Bullfinch Rd, Panama City Beach, FL 32407 (imelda.r.muller@gmail.com). doi:10.12788/cutis.0033 


\section{THE DIAGNOSIS: Neutrophilic Dermatosis of the Dorsal Hands}

$\mathrm{M}$ icroscopic specimen analysis demonstrated epidermal ulceration, a diffuse dermal neutrophilic infiltrate, and papillary edema (Figure) consistent with neutrophilic dermatosis of the dorsal hands $(\mathrm{NDDH})$. Special stains and cultures were negative for bacterial and fungal organisms. The patient was treated with high-dose oral prednisone $80 \mathrm{mg}$ daily for 1 week (tapered over the course of 7 weeks) and dapsone gel 5\% twice daily with rapid wound resolution. An extensive review of systems, age-appropriate malignancy screening, and laboratory evaluation did not demonstrate underlying systemic illness, infection, or malignancy.

Neutrophilic dermatosis of the dorsal hands commonly arises alongside traumatic injury and presents as a nonhealing hand wound. ${ }^{1}$ It is considered a localized variant of acute febrile neutrophilic dermatosis (Sweet syndrome), a systemic inflammatory condition characterized by fever, malaise, neutrophilia, and elevated inflammatory markers. ${ }^{1,2}$ Cutaneous lesions are variable and may include pustular nodules; tender, purulent, violaceous plaques with ulceration and crusting; or hemorrhagic bullae resembling coagulopathy or an infectious etiology. ${ }^{1,3}$ Leukocytoclastic vasculitis may present with bullous or ulcerative lesions and also histologically resembles NDDH. ${ }^{4}$ Although ulceration typically is not common in Sweet syndrome, the ulcerated lesions with elevated, edematous, and violaceous borders in our patient were characteristic of NDDH.

Neutrophilic dermatosis of the dorsal hands, similar to Sweet syndrome, may arise along with malignancy, infection (eg, respiratory, gastrointestinal, hepatitis C virus), systemic illnesses (eg, inflammatory bowel disease, colitis, rheumatoid arthritis, Raynaud phenomenon), or environmental exposure (eg, fertilizer) or with the use of certain medications (eg, thalidomide, minocycline).$^{1-3,5}$ Both solid tumors (eg, breast and lung carcinomas) as well as hematologic disturbances (eg, leukemia, myelodysplasia, lymphoma) have been associated with $\mathrm{NDDH} .^{1-3}$ Although NDDH appears to be idiopathic, all patients should undergo an extensive review of systems, laboratory evaluation, and age-appropriate malignancy screening.

Given the rarity of NDDH, necrotic lesion appearance, and potential for secondary infection, patients often are misdiagnosed with infectious etiologies, including necrotizing fasciitis. . $1,3,67$ Lesions of blastomycosislike pyoderma also may be pustular or ulcerative with elevated borders resembling NDDH. ${ }^{8}$ The pathogenesis of this rare condition remains uncertain. Although systemic antibiotics are a commonly utilized treatment modality, their efficacy may be primarily related to their anti-inflammatory properties. ${ }^{8}$
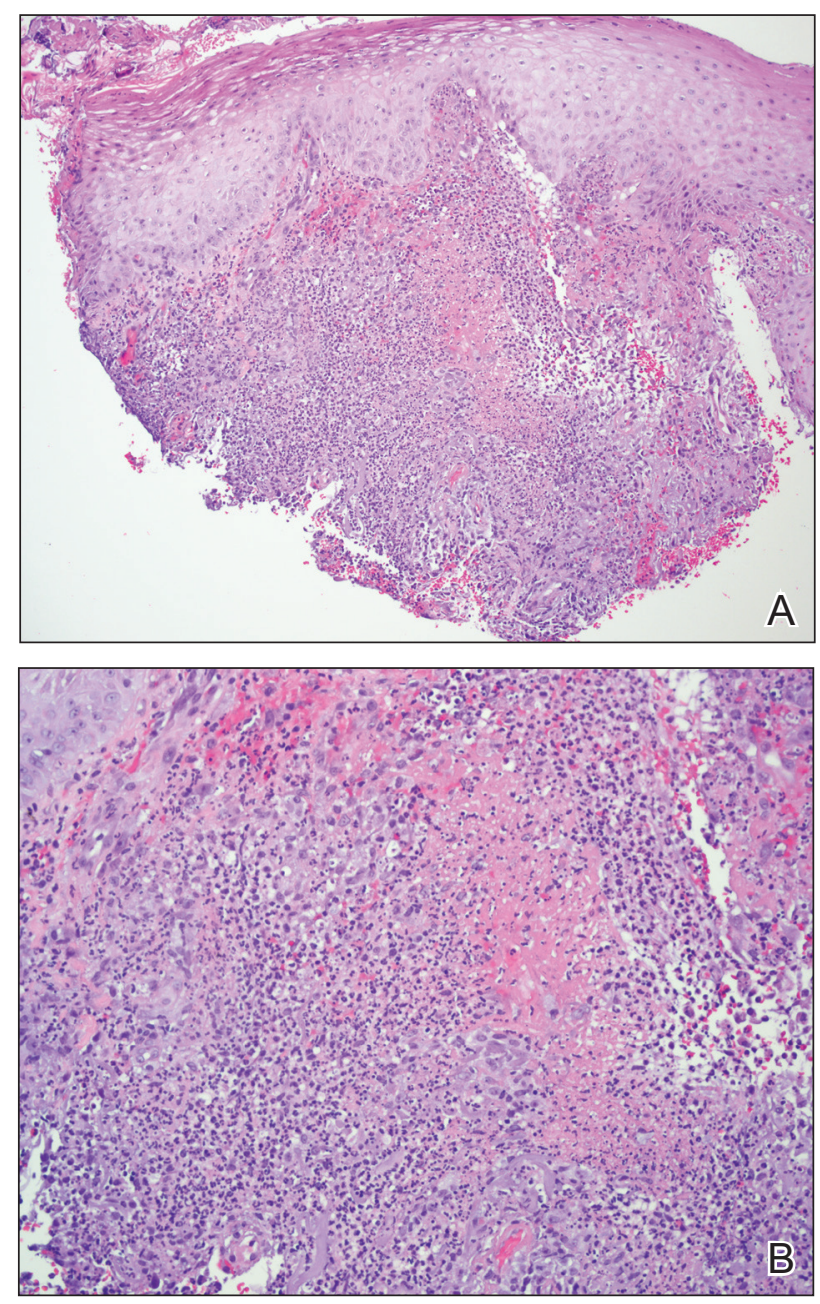

A and B, Neutrophilic dermatosis of the dorsal hands. Diffuse neutrophilic infiltrate and fibrinoid debris (H\&E, original magnifications $\times 100$ and $\times 200$ ).

Mycobacterium marinum is an aquatic nontuberculous mycobacterium that causes ulcerated, nodular, or pustular cutaneous granulomas that may resemble the lesions of NDDH. ${ }^{9}$ Similar to NDDH, lesions develop in areas of minor skin trauma, often on the upper extremities. At-risk individuals include those in frequent contact with aquatic environments, lending to the term fish tank granuloma. Diagnosis is made through culture, tissue biopsy, or the presence of acid-fast bacilli. Antibiotics such as doxycycline, surgical debridement, or cryotherapy are effective treatments. ${ }^{9}$

Unlike infectious etiologies of similarly appearing lesions, primary lesions of NDDH are aseptic. Treatment with antibiotics is ineffective, and surgical intervention can result in devastating expansion of existing wounds as well 
PHOTO CHALLENGE DISCUSSION

as development of new lesions at surgical margins due to the pathergy effect and Koebner phenomenon. ${ }^{3,6}$ The intiation of systemic corticosteroids and/or dapsone results in prompt resolution of $\mathrm{NDDH} .{ }^{1}$ In recalcitrant cases or when steroids are contraindicated, other medications may be used including dapsone, colchicine, potassium iodide, indomethacin, or biologics. ${ }^{2}$

Atypical pyoderma gangrenosum is a bulbous variant of pyoderma gangrenosum that is clinically and histologicoly indistinguishable from NDDH. ${ }^{2,10}$ Atypical pyoderma gangrenosum frequently presents on the upper extremeties, exhibits a pathergy response to trauma, is associated with similar systemic diseases, and is treated identically to NDDH. There is some degree of uncertainty about the classification and pathophysiology of atypical pyoderma gangrenosum, NDDH, and Sweet syndrome. The compelling similarities may indicate that these cutaneous disorder represent a spectrum of the same disease. ${ }^{2,10}$

Consideration of NDDH in the differential of nonhealing hand wounds is paramount to prevent progression and iatrogenic morbidity associated with delayed and missed diagnosis. Early recognition of NDDH may allow for earlier diagnosis of frequently associated systemic illnesses and malignancies.
REFERENCES

1. DiCaudo DJ, Connolly SM. Neutrophilic dermatosis (pustular vasculitis) of the dorsal hands: a report of 7 cases and review of the literature. Arch Dermatol. 2002;138:361-365.

2. Walling HW, Snipes CJ, Gerami P, et al. The relationship between nutrophilic dermatosis of the dorsal hands and Sweet syndrome: report of 9 cases and comparison to atypical pyoderma gangrenosum. Arch Dermatol. 2006;142:57-63.

3. Chen AMY, Chen HS, Smith BJ, et al. Neutrophilic dermatosis of the hands: a review of 17 cases. J Hand Surg Am. 2018; 43:185.E1-185.E5.

4. Russell JP, Gibson LE. Primary cutaneous small vessel vasculitis: approach to diagnosis and treatment. Int J Dermatol. 2006;45:3-13.

5. Gaur S, Gupta D, Gary B, et al. Neutrophilic dermatosis of the dorsal hands. Indian Dermatol Online J. 2015;6:42-45.

6. Cooke-Norris RH, Yous JS, Gibson LE. Neutrophilic dermatosis of the hands: an underrecognized hematological condition that may result in unnecessary surgery. Am J Hematol. 2009;84:60-61.

7. Kroshinsky D, Allow A, Rothschild B, et al. Necrotizing Sweet syndrome: a new variant of neutrophilic dermatosis mimicking necrotizing fasciitis. J Am Acad Dermatol. 2012;67:945-954.

8. Hongal AA, Gejje S. Blastomycosis-like pyoderma-a rare case report. J Chin Dian Res. 2016;10:WD03-WD04.

9. Petrine B. Mycobacterium marinum: ubiquitous agent of waterborne granulomatous skin infections. Eur J Chin Microbiol Infect Dis. 2006;25:609-613.

10. Ahronowitz I, Harp J, Shinkai K. Etiology and management of yoderma gangrenosum: a comprehensive review. Am J Chin Dermatol. 2012;13:191-211.

WW.MDEDGE.COM/DERMATOLOGY

VOL. 106 NO. 2 I AUGUST 202075

Copyright Cutis 2020. No part of this publication may be reproduced, stored, or transmitted without the prior written permission of the Publisher. 